\title{
Scene Simulation of Emergency Drills for Outpatient Unexpected Accidents and the Effects on Patients with HAMD, SAS Scores
}

\author{
Fan Yanmei, Chen yang, Wang Danting, Li Jie, Zhang Xiuli, Li Xuantong \\ The Second Affiliated Hospital of Xi'an Jiaotong University, Xi'an, China
}

Email address:

2912520612@qq.com (Fan Yanmei)

\section{To cite this article:}

Fan Yanmei, Chen yang, Wang Danting, Li Jie, Zhang Xiuli, Li Xuantong. Scene Simulation of Emergency Drills for Outpatient Unexpected Accidents and the Effects on Patients with HAMD, SAS Scores. Science Innovation. Vol. 7, No. 6, 2019, pp. 171-174. doi: $10.11648 /$ j.si.20190706.14

Received: August 21, 2019; Accepted: December 23, 2019; Published: December 26, 2019

\begin{abstract}
Objective: To study the effect of scene simulation emergency drill on unexpected accidents in outpatient department and its influence on Hamilton Depression Scale (HAMD) and Self rating Anxiety Scale (SAS) score. Methods: 40 cases of hospital nurses as the research object, randomly divided into study group and control group, 20 cases in each group. The control group was given routine nursing training, study group underwent simulation training. Emergency drills were compared before and after training of two groups of nurses' comprehensive ability. At the same time, the study group and the control group of 100 cases were selected in our hospital outpatient emergency analysis of the training effect. Compared with two groups of patients with emergency response time, HAMD, SAS score and satisfaction. Results: After training the study nurse a clear division of labor, reasonable position, quick action, regulations clear, correct operation were significantly higher than control group $(P<0.05)$. The study group of condition evaluation time, establish vein channel check time, specimen time, resuscitation room rescue time were significantly lower than the control group (all $P<0.05)$.After training, the HAMD and SAS scores of the study group were $(21.34 \pm 4.76),(32.22 \pm 5.44)$, which were significantly lower than that of the control group $(28.92 \pm 5.15),(41.64 \pm 6.13)$ (all $P<0.05)$.After the training study group patients satisfaction was $100 \%(100 / 100)$, significantly higher than the control group 93\% $(93 / 100)(P<0.05)$. Conclusion: Simulated emergency drills can effectively improve the comprehensive ability of nurses, and effectively shorten the processing time of outpatient sudden accidents, relieve patients anxiety, depression and other negative emotions, improve patient satisfaction.
\end{abstract}

Keywords: Outpatient Emergency Accidents, Scenario Simulation Emergency Exercise, Hamilton Depression Scale, Self Rating Anxiety Scale, Satisfaction Degree

\section{情景模拟急救演练对门诊突发意外事件的作用及对患者HAMD、SAS 评分的影响}

范艳梅*, 陈杨, 王丹婷, 李洁, 张秀丽, 李炫同

西安交通大学第二附属医院门诊部, 西安, 中国

邮箱

2912520612@qq. com（范艳梅）

摘要：目的：研究情景模拟急救演练对门诊突发意外事件的作用及对患者汉密尔顿抑郁量表（HAMD）、焦虑自评量表 (SAS) 评分的影响。方法: 选取我院40例护士为研究对象, 以随机数字表法均分为研究组与对照组, 每组各20例。对 照组予以常规护理培训, 研究组则行情景模拟急救演练培训。分别比较培训前后两组护士综合能力情况。同时, 研究 
组与对照组分别选取 100 例于我院门诊急救的患者分析培训效果。比较两组急救反应时间, 患者HAMD、SAS评分以及满 意度情况。结果：培训后研究组护士分工明确、合理站位、动作迅速、条例清楚、操作正确评分均明显高于对照组 (均 $P<0.05$ ）。研究组病情评估时间、建立静脉通道时间、检查标本送检时间、抢救室抢救时间均明显低于对照组（均 $P$ $<0.05)$ 。培训后研究组患者HAMD、SAS评分分别为 (21.34 44.76) 分、(32.22 25.44$)$ 分, 均明显低于对照组的 (28.92 $\pm 5.15 ）$ 分、 (41.64 $\pm 6.13 ）$ 分（均 $\mathrm{P}<0.05 ）$ 。培训后研究组患者满意度为 $100.00 \% （ 100 / 100 ）$, 明显高于对照组 的93.00\%（93/100）（ $P<0.05 ）$ 。结论: 情景模拟急救演练可有效提高门诊护士综合能力, 同时有效缩短门诊突发意 外事件的处理时间，明显缓解患者焦虑、抑郁等负性情绪，提高患者满意度。

关键词：门诊突发意外事件，情景模拟急救演练，汉密尔顿抑郁量表，焦虑自评量表，满意度

\section{1. 引言}

门诊是医院中患者集中就诊的场所, 具有疾病种类不一、 可预见性差以及突发意外事件风险较高的特点 [1]。而门诊护 士受多种因素影响, 学习积极性较差, 急救相关知识以及技 能较弱, 在碰到突发意外事件时的急救抢救能力明显贵乏[2]。 从而可能影响门诊患者的急救效果, 进一步导致医疗纠纷事 件的发生。因此, 寻找一种有效的培训方式, 对改善门诊护 士的应对突发事件能力显得无为重要。鉴于此, 本文通过研 究情景模拟急救演练对门诊突发意外事件的作用及对患者 汉密尔顿抑郁量表（HAMD）、焦虑自评量表（SAS）评分 的影响, 旨在为临床治疗提供数据支持, 现作如下报道。

\section{2. 资料与方法}

\section{1. 一般资料}

选取我院 40 例护士为研究对象, 以随机数字表法均分为 研究组与对照组, 每组各20例。研究组年龄21 52岁, 平均 年龄 $(28.4 \pm 3.4)$ 岁; 从事门诊护理工作时间 6 个月 15 年, 平均从事门诊护理工作时间 $(6.5 \pm 2.3)$ 年; 受教育程度: 中 专学历 8 例, 专科学历 11 例, 本科学历 1 例。对照组年龄 22 51 岁, 平均年龄 $(28.2 \pm 3.3)$ 岁; 从事门诊护理工作时间 8 个月 15 年, 平均从事急诊护理工作时间 $(6.6 \pm 2.3)$ 年; 受 教育程度: 中专学历7例, 专科学历 11 例, 本科学历 2 例。两 组护士年龄、从事门诊护理工作时间和受教育程度相比 (P $>0.05$ ）, 说明组间具有可比性。同时，研究组2016年7月 1 日-2018年12月31日与对照组2015年1月1日-2016年7月1日分 别选取 100 例于我院门诊急救的患者 100 例分析培训效果。其 中研究组男性患者 67 例, 女性患者33例, 年龄 $18 \sim 72$ 岁, 平 均年龄 $(43.3 \pm 10.4)$ 岁; 疾病类型: 心源性猝死18例, 脑出 血31例, 低血糖22例, 高血压15例, 其他16例。对照组男性 患者 65 例, 女性患者 35 例, 年龄 $18 \sim 74$ 岁, 平均年龄

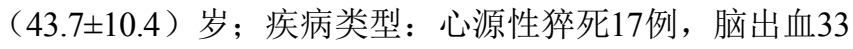
例, 低血糖20例, 高血压17例, 其他15例。两组患者性别, 年龄与疾病类型比较, $(\mathrm{P}>0.05)$, 说明组间存在可比性。 两组患者均签署了知情同意书, 我院伦理委员会已批准。

\section{2. 研究方法}

对照组予以常规护理培训, 研究组则行情景模拟急救 演练培训, 具体内容如下:

\subsection{1. 制定预案流程}

参照由美国心脏协会发布的《2010版心肺复苏指南》 以及相关危急重症急救流程, 制定门诊突发意外事件抢 救预案和流程。预案主要是以就地救治为原则, 一旦患 者突发病情时, 立即由现场附近的医护人员就地开展第 一时间的抢救, 明确责任医师、护士, 紧急联系的方式。 同时, 按照门诊不同科室的特征, 设计急救护理模拟情 景, 主要包括呼吸心脏骤停、晕厥以及迟缓型药物过敏 性休克等。

\subsection{2. 培训考核}

培训内容主要与急救相关知识为主, 包括《2010版心 肺复苏指南》、门诊突发意外事件抢救预案和流程、各项 护理核心制度等, 由护士长或带教老师负责。培训方式以 理论授课和技术操作训练结合为主, 定期每年组织 2 次理 论知识以及技能的培训、考核, 且规定每年的 1 月和 7 月的 首周为培训周，末周为考核周，共培训、考核2次。

\subsection{3. 演练步骤}

每次演练模拟 1 个急救场景, 可选择在候诊大厅、检 验窗口、心内科诊区以及卫生间等地方, 演练时间尽量选 择在门诊患者较少的下午时段。演练前做好告示, 演练时 则由护士长以及带教老师带队, 并担任评委。

\subsection{4. 总结反馈}

演练结束后组织护士进行现场反馈, 并由评委进行 讲评, 明确演练过程中存在的一系列问题, 并提出改进 建议与措施。最后, 组织护士观看演练录像, 结合现场 反馈的情况对演练进行推敲, 落实改进措施, 反复优化 救治流程。

\subsection{5. 观察指标}

分别比较培训前后两组护士综合能力情况, 两组患 者急救反应时间, HAMD、SAS评分以及满意度情况。 其中护士综合能力主要是通过综合能力考评结果进行评 定, 主要包括分工明确、合理站位、动作迅速、条例清 楚、操作正确五项, 每项得分 $1 \sim 5$ 分, 得分越高表示护 士综合能力越高[3]。汉密尔顿抑郁量表 (HAMD) 主要 包括 24 个项目, 其中 10 个项目得分为 $1 \sim 2$ 分, 14 个项目 得分 $1 \sim 4$ 分, 总分 76 分, 得分越高表示患者抑郁情况越 严重[4]。焦虑自评量表（SAS）包含20个项目, 其中每 
个项目得分 $1 \sim 4$ 分, 总分 $20 \sim 80$ 分, 得分越高表示患者 焦虑程度越严重 [5]。采用满意度测评量表对患者满意度 进行调查评估 [6]: 主要包括医务人员热情与否、医务人 员仪表端庄和热情服务与否、医务人员技能操作熟练与 否、医务人员指导服药与否等 10 个问题, 每个问题均有 是、一般以及否三个选项, 其中选择给 10 分, 选一般给 5 分, 选择否给 0 分。（1）十分满意：总得分为 $90 \sim 100$ 分; (2) 较满意: 总得分为 $60 \sim 90$ 分, (3) 不满意: 总得分 $<60$ 分。满意度 $=$ (十分满意人数+较满意人数) / 总人数 $\times 100 \%$ 。

\section{3. 统计学方法}

本研究数据均采用SPSS20.0软件进行检测分析, 采用 “ $\chi^{2}$ ”检验、 $\mathrm{t}$ 检验, 用“[n(\%)]”表示计数资料, 用“ $\left(\bar{x}_{ \pm \mathrm{s}}\right)$ ”
表示计量资料均数标准差。 $P$ 值 $<0.05$ 表明两组数据对比 具有统计学意义。

\section{3. 结果}

\section{1. 培训前后两组护士综合能力对比}

培训后研究组护士分工明确、合理站位、动作迅速、 条例清楚、操作正确评分分别为 $(4.13 \pm 0.82)$ 分、(4.73 \pm 0.38$)$ 分、 $(4.71 \pm 0.42)$ 分、 $(4.56 \pm 0.52)$ 分、 $(4.51 \pm 0.62)$ 分, 均明显高于对照组的 $(3.58 \pm 0.79)$ 分、 (3.94 \pm 0.72$)$ 分、 $(4.03 \pm 0.76)$ 分、 $(3.90 \pm 0.77)$ 分、 $(3.57 \pm 1.02)$ 分 (均 $P<0.05)$ 。见下表1:

表1 培训前后两组护士综合能力对比 (分, $\bar{x} \pm \mathrm{s}$ )。

\begin{tabular}{llllll}
\hline 组别 & 时间 & 研究组 $(\mathbf{n}=\mathbf{2 0})$ & 对照组 $(\mathbf{n}=\mathbf{2 0})$ & $\mathbf{t}$ 值 & P值 \\
\hline 分工明确 & 培训前 & $3.01 \pm 0.72$ & $3.02 \pm 0.74$ & 0.097 & 0.923 \\
& 培训后 & $4.13 \pm 0.82^{*}$ & $3.58 \pm 0.79$ & 4.830 & 0.000 \\
合理站位 & 培训前 & $3.84 \pm 0.68$ & $3.79 \pm 0.63$ & 0.539 & 0.590 \\
& 培训后 & $4.73 \pm 0.38^{*}$ & $3.94 \pm 0.72$ & 9.704 & 0.000 \\
动作迅速 & 培训前 & $3.78 \pm 1.14$ & $3.82 \pm 1.15$ & 0.247 & 0.805 \\
& 培训后 & $4.71 \pm 0.42^{*}$ & $4.03 \pm 0.76$ & 7.831 & 0.000 \\
条例清楚 & 培训前 & $3.68 \pm 1.21$ & $3.65 \pm 1.18$ & 0.178 & 0.859 \\
操作正确 & 培训后 & $4.56 \pm 0.52^{*}$ & $3.90 \pm 0.77$ & 7.103 & 0.000 \\
\hline
\end{tabular}

注：与对照组相比, $* \mathrm{P}<0.05$ 。

\section{2. 两组急救反应时间情况对比}

间、抢救室抢救时间(15.38 \pm 5.27 vs 29.46 \pm 5.73$)$ 均明显低于

研究组病情评估时间（3.08 \pm 1.08 vs 4.82 $\pm 1.74 ）$ 、建 立静脉通道时间 $(2.87 \pm 1.67$ vs 3.56 \pm 2.18$)$ 、检查标本送检时

表2 两组急救反应时间情况对比（ $\min , \bar{x} \pm \mathrm{s})$ 。

\begin{tabular}{|c|c|c|c|c|}
\hline 组别 & 例数 & 病情评估时间 & 静脉通路通路时间 & 抢救室抢救时间 \\
\hline 研究组 & 100 & $3.08 \pm 1.08 *$ & $2.87 \pm 1.67^{*}$ & $15.38 \pm 5.27^{*}$ \\
\hline 对照组 & 100 & $4.82 \pm 1.74$ & $3.56 \pm 2.18$ & $29.46 \pm 5.73$ \\
\hline t值 & - & 8.496 & 2.513 & 18.086 \\
\hline P值 & - & 0.000 & 0.013 & 0.000 \\
\hline
\end{tabular}

注: 与对照组相比, $* \mathrm{P}<0.05$

\section{3. 培训后两组患者HAMD、SAS评分对比}

培训后研究组患者 HAMD、SAS 评分分别为 (21.34 \pm 4.76$)$ 分、 (32.22 \pm 5.44$)$ 分, 均明显低于对照组 的 $(28.92 \pm 5.15)$ 分、 $(41.64 \pm 6.13)$ 分 (均 $P<0.05)$ 。 见下表3:

表3 培训后两组患者HAMD、SAS评分对比（分, $\bar{x} \pm \mathrm{s}$ )。

\begin{tabular}{llll}
\hline 组别 & 例数 & HAMD & SAS \\
\hline 研究组 & 100 & $21.34 \pm 4.76^{*}$ & $32.22 \pm 5.44$ \\
对照组 & 100 & $28.92 \pm 5.15$ & $41.64 \pm 6.13$ \\
t值 & - & 10.809 & 11.494 \\
P值 & - & 0.000 & 0.000 \\
\hline
\end{tabular}

注：与对照组相比, $* \mathrm{P}<0.05$ 。

\section{4. 培训后两组患者满意度情况对比}

培训后研究组患者满意度为 $100.00 \%(100 / 100)$, 明显高于对照组的 $93.00 \%(93 / 100) （ P<0.05 ）$ 。见下 表4:

表4 培训后两组患者满意度情况对比（例，\%）。

\begin{tabular}{llllll}
\hline 组别 & 例数 & 十分满意 & 较满意 & 不满意 & 满意度 \\
\hline 研究组 & 100 & 73 & 27 & 0 & $100(100.00)^{*}$ \\
对照组 & 100 & 65 & 28 & 7 & $93(93.00)$ \\
$\chi^{2}$ 值 & - & - & - & - & 7.254 \\
$\mathrm{P}$ 值 & - & - & - & - & 0.007 \\
\hline
\end{tabular}

注: 与对照组相比, $* \mathrm{P}<0.05$ 。 


\section{4. 讨论}

门诊护理具有突发性、复杂性以及多变性等特点，特别 是突发意外事件时，综合医院门诊急危重患者多，工作节奏 较快, 因此对护士的综合护理能力具有较高的要求[7-8]。因 此，如何有效提高护理人员的综合能力，对提高医院护理质 量, 改善患者预后具有极其重要的意义。而情景模拟演练主 要是指通过设计一种逼真的工作场景或管理系统, 要求被培 训者根据一定的要求完成相应的任务, 从中锻炼以及考察其 工作能力的一种方式[9-10]。本研究结果显示: 培训后研究 组护士分工明确、合理站位、动作迅速、条例清楚、操作正 确评分均明显高于对照组。这与朱紫英等人的研究报道相一 致[11-12], 说明了情景模拟急救演练培训可有效提高门诊护 士的综合能力。其中主要原因在于: 情景模拟急救演练有利 于让护士在实际工作中将理论知识与实践相结合, 通过情景 模拟急救演练发现抢救过程中所存在的不足之处, 从而进行 不断改进, 并通过反复情景模拟急救演练, 有利于护士明确 常规急救流程, 帮助其掌握在应对突发意外事件时应采取的 相应急救流程, 从而有效保证在最短的时间内挽救患者生命, 进一步缩短了理论与实践的距离, 提高护士的急救综合能力。 此外, 研究组病情评估时间、建立静脉通道时间、抢救室抢 救时间均明显低于对照组。这提示了情景模拟急救演练培训 可有效缩短急救时间。分析原因, 情景模拟急救演练为护士 提供了紧张的工作氛围, 从而有效锻炼护士的心理素质, 帮 助其在应对突发意外事件时, 可快速对病情进行判断以及评 估; 与此同时，该培训方式有利于提高护士在实际抢救工作 中熟练开展一系列应激工作, 并准确地实施相关操作, 为危 急重症患者的抢救争取了一定的时间。另外, 培训后研究组 患者HAMD、SAS评分均明显低于对照组。这提示了情景模 拟急救演练培训有效缓解患者焦虑、抑郁等负面情绪。究其 原因, 门诊患者通常会存在不同程度的情绪变化, 加之对疾 病以及治疗方式未知会引发紧张、恐惧等情绪, 进一步加剧 了患者的心理问题。而情景模拟急救演练培训要求护士关注 患者及其家属的情绪变化, 并与其进行积极沟通交流, 为其 提供人性化护理，从而有效缓解患者的负面情绪。同时，培 训后研究组患者满意度明显高于对照组[13], 这说明了情景 模拟急救演练可显著提高患者满意度。其中主要原因是门诊 急救患者及家属普遍存在不同程度的急切救治心理, 加上病 情的复杂性与不确定性, 家人通常会寄希望于护士身上, 而 护士姻熟的抢救技术以及有条不紊的抢救流程提高了患者 的信任度与依赖性, 从而提高了满意度。

\section{5. 结论}

情景模拟急救演练可有效提高门诊护士综合能力, 同 时有效缩短门诊突发意外事件的处理时间, 明显缓解患者
焦虑、抑郁等负面情绪, 提高患者满意度。值得临床推广 应用。

\section{参考文献}

[1] 王淑娟,孙丽娜,康岩,等.循证护理在急诊科情景模拟急救演 练中的应用 [J].护理研究,2015,29(10):3699-3701。

[2] 王荣英, 李峰,贺振银, 等.三级综合医院简易门诊慢性病患者 就诊特点和满意度分析 [J]. 中华全科医师杂 志,2017,16(09):687-691。

[3] 赵风军,赵秀荣.医护一体化责任制合作模式对提高护士综 合护理能力的影响 [J]. 中华现代护理杂 志,2014,20(26):3302-3306。

[4] 刘明勇,周立春.特发性良性阵发性位置性眩军患者发病前 焦虑抑郁情况的调查 [J]. 中华神经科杂 志,2017,50(05):358-361。

[5] 余爵波,程泽星,郑海洲,等.老年变应性鼻炎患者生活质量和 焦虑抑郁情绪症状分析 [J]. 中华老年医学杂 志,2017,36(08):895-898。

[6] 许欣悦,田玮,李卫红,等.北京市某三级甲等医院知名专家团 队层级转诊患者满意度调查 [J]. 中华医院管理杂 志,2017,33(06):413-415。

[7] 杨春风,刘杰,顾春光,等.急救情景演练提升社区急救能力探 索 $[J]$.上海医药,2015,36(24):17-18。

[8] 徐加萍,吕君.情景模拟演练对急诊多发性创伤病人救治效 果的影响[J].全科护理,2016,14(33):3515-3616。

[9] 王倩,谭焯筠,刘莉,等.情景模拟教学法在ICU低年资护士应 急能力培训中的应用 [J]. 实用临床护理学电子杂 志,2017,02(06):163-163。

[10] 胡静.情景模拟演练在培养急诊低年资护士抢救应急能力 中的应用[J].检验医学与临床,2017,14(16):2489-2491。

[11] 朱紫英.情景模拟急救演练提高门诊护士应对意外事件中 的作用 $[\mathrm{J}]$.上海护理, 2015,15(02):86-88。

[12] 田雪,张楠,罗曼,等.情景模拟教学法在口腔门诊护士急救技 能培训中的应用[J].贵阳中医学院学报,2015,37(05):72-74。

[13] Frick J, Möckel M, Muller R,et al.Suitability of current definitions of ambulatory care sensitive conditions for research in emergency department patients: a secondary health data analysis[J].BMJ Open,2017,7(10):16109-16110。 